\title{
7. \\ Die Krümmungslinie der Wellenfläche zweiaxiger Krystalle.
}

(Von Herrn P. Zech in Stultgart.)

(Im 52. Bande dieses Journals habe ich die Eigenschaften der Wellenfläche zweiaxiger Krystalle untersucht, welche sich aus der punktweisen Construction derselben ergeben. Eine Reihe weiterer Eigenschaften findet man, wenn man sie als Einhüllungsfläche von Ebenen betrachtet. Ich bediene mich dabei derselben Bezeichnungen, wie in jenem Aufsatz und fahre in der Nummernzahl fort.)

\section{9.}

Zu den Mantellinien eines Kegels $\boldsymbol{K}$, welcher die optischen Axen des Ellipsoids $\boldsymbol{E}$ zu Fokallinien hat, lege man senkrechte Ebenen im Abstand der reciproken Mantellinienlänge des Ergänzungskegcls $\boldsymbol{C}$ vom Mittelpunkt $\boldsymbol{O}$. Diese Ebenen sind Berührungsebenen an die Wellenfläche, ihre Einhüllungsfläche ist eine entwickelbare Fläche $\boldsymbol{F}$ : sie ist einer Kugel mit dem Mittelpunkt $\boldsymbol{O}$ und mit einem Halbmesser gleich jener reciproken Länge umschrieben und ihre Berührungsebenen sind den Berührungsebenen des Ergänzungskegels $\boldsymbol{C}$ parallel, oder sie ist die Einhüllungsfläche der gemeinschaftlichen Berührungsebenen jener Kugel und des Kegelschnitts, in welchem $\boldsymbol{C}$ die unendlich ferne Ebene schneidet (unter Berührungsebene eines Kegelschnitts ist jede Ebene zu verstehen, welche durch eine seiner Tangenten geht). Die Fläche $\boldsymbol{F}$ hat dieselben Hauptschnittebenen wie die Wellenfläche und ist durch zwei auf verschiedenen Hauptschnittebenen senkrechte Berührungsebenen bestimmt. Die Erzeugenden der Fläche $\boldsymbol{F}$ liegen in den Normalebenen des Kegels $\boldsymbol{K}$, weil je zwei aufeinanderfolgende Berührungsebenen von $\boldsymbol{F}$ senkrecht stehen auf zwei aufeinanderfolgenden Mantellinien von $\boldsymbol{K}$. Die Wellenfläche ist die Einhüllungsfläche aller möglichen entwickelbaren Flächen $\boldsymbol{F}$, welche den verschiedenen Kegeln $\boldsymbol{K}$ entsprechen.

Es giebt noch eine zweite Art entwickelbarer Flächen, als deren Einhüllungsfläche die Wellenfläche sich betrachten läfst. Ist $O N$ Mantellinie eines Kegels $K, O S$ die entsprechende Mantellinie des Ergänzungskegels $\boldsymbol{C}$ (2.) 
oder die eine Halbaxe des auf $\boldsymbol{O N}$ senkrechten Diametralschnitts von $\boldsymbol{E}, \boldsymbol{O Q}$ die zweite Halbaxe dieses Schnitts, so sind zwei zu $O N$ senkrechte Ebenen in den $A$ bständen $\frac{1}{O S}$ und $\frac{1}{O Q}$ von $O$ Berührungsebenen an die Wellenfläche. Alle möglichen zu den Mantellinien von $\boldsymbol{K}$ senkrechte Ebenen im constanten Abstand $\frac{1}{O S}$ von $O$ bilden eine Fläche $\boldsymbol{F}$, alle möglichen zu den Mantellinien von $K$ senkrechte Ebenen in dem veränderlichen Abstand $\frac{1}{O Q}$ von $O$ bilden eine entwickelbare Fläche $\boldsymbol{G}$, welche demselben unendlich fernen Kegelschnitt wie $\boldsymbol{F}$ umschrieben ist. Ist nun $O V$ der Abstand einer zu $O N$ sankrechten Berührungsebene an $E$ von $O$, so ist $O V . O S . O Q$ constant als Inhalt eines Parallelepipeds aus drei conjugirten Halbmessern von $\boldsymbol{E}$, welche Richtung auch $\boldsymbol{O N}$ habe: bleibt $\boldsymbol{O N}$ auf $K$, so ist $O S$ constant, also auch $O V . O Q$, oder das Verhältnifs $O V: \frac{1}{O Q}$, d. h. die Berührungsebenen der Fläche $G$ sind Berührungsebenen an ein $\boldsymbol{E}$ ähnliches Ellipsoid. Die Fläche $\boldsymbol{G}$ ist also die Einhüllungsfläche der gemeinschaftlichen Berührungsebenen an ein $\boldsymbol{E}$ ähnliches Ellipsoid und an einem unendlich fernen Kegelschnitt; sie hat dieselben Hauplschnittebenen wie die Wellenfläche, ihre Erzeugenden liegen in den Normalebenen des Kegels $\boldsymbol{K}$. Die Wellenfläche ist die Einhüllungsfläche aller möglichen Flächen $\boldsymbol{G}$. Jede Fläche $\boldsymbol{G}$ ist hestimmt, sobald man von ihr zwei zu verschiedenen Hauptschnittebenen senkrechte Berührungsebenen kennt.

Sind $\boldsymbol{K}_{1}$ und $\boldsymbol{K}_{2}$ zwei sich schneidende Kegel, welche die optischen Axen von $\boldsymbol{E}$ zu Fokallinien haben, so bestimmt der Kegel $\boldsymbol{K}_{1}$ eine Fläche $\boldsymbol{F}_{1}$ und eine Fläche $G_{1}$, welche demselben unendlich fernen Kegelschnitt umschrieben sind, der Kegel $\boldsymbol{K}_{2}$ eine Fläche $\boldsymbol{F}_{2}$ und eine Fläche $\boldsymbol{G}_{2}$, welche auch Einem unendlich fernen Kegelschnitt umschrieben sind. Die zu einer gemeinschaftlichen Mantellinie von $\boldsymbol{K}_{1}$ und $\boldsymbol{K}_{2}$ senkrechten Berührungsebenen an die Wellenfläche sind Berührungsebenen der 4 Flächen $\boldsymbol{F}_{1}, \boldsymbol{G}_{1}, \boldsymbol{F}_{2}, \boldsymbol{G}_{2}$ und zwar die eine von $\boldsymbol{F}_{1}$ und $\boldsymbol{G}_{2}$, die andere von $\boldsymbol{F}_{2}$ und $\boldsymbol{G}_{1}$. Jede Berührungsebene der Wellenfläche läfst sich also als Berührungsebene entweder einer Fläche $\boldsymbol{G}$ oder einer Fläche $\boldsymbol{F}$ betrachten; die zwei Flächen sind bestimmt durch sich rechtwinklig schneidende Kegel, also sind die Erzeugenden, längs welcher sie von jener Berührungsebene berührt werden, senkrecht auf einander, weil die eine $\cdot$ in der Normalebene eines Kegels $\boldsymbol{K}_{1}$, die andere in der Normalebene eines Kegels $\boldsymbol{K}_{2}$ längs ihrer gemeinschaftlichen Mantellinie liegt. 


\section{0.}

Die zweị Arten entwickelbarer Flächen $F$ und $G$ sind die Polarflüchen der ellipsoidischen und sphärischen Kegelschnitte auf der Wellenfläche in Beziehung auf ein Ellipsoid $D$, welches dieselben Hauptschnittebenen wie die Wellenfläche und die Halbaxen $\sqrt{b c}, \sqrt{c a}, \sqrt{a b}$ hat, wenn die des Ellipsoids $₹$ beziehungsweise nach denselben Richtungen $a, b$ und $c$ sind.

Denn die Polarfläche eines ellipsoidischen Kegelschnitts ist die Einhüllungsfläche der gemeinschaftlichen Berührungsebenen der Polarflächen des Ellipsoids und des Kegels, die sich längs des Kegelschnitts schneiden. Das Ellipsoid ist $E$ ähnlich (6.), hat also die Halbaxen $\frac{1}{m a}, \frac{1}{m b}, \frac{1}{m c}$, wo $m$ verschieden ist für verschiedene ellipsoidische Kegelschnitte; seine Polarfläche für das Ellipsoid $\boldsymbol{D}$ ist ein Ellipsoid mit den Halbaxen mabc, mbca, mcab, d. b. eine Kugel. Die Polarfläche des Kegels ist durch einen unendlich fernen Kegelschnitt vorgestellt. Die Polarfläche jedes ellipsoidischen Kegelschnitts ist also eine einer Kugel und einem unendlich fernen Kegelschnitt umschriebene entwickelbare Fläche, und daher durch zwei auf verschiedenen Hauptschnittebenen der Wellenfläche senkrechte Berührungsebenen bestimmt. Die vier Scheitel des ellipsoidischen Kegelschnitts liegen auf zwei Kreisen, in welchen die Wellenfläche zwei ihrer Hauptschnittebenen schneidet; die Polarflächen dieser Kreise für $\boldsymbol{D}$ sind elliptische Cylinder, welche, wie sich leicht ergiebt, die Wellenfläche längs der zwei Ellipsen berühren, in welchen die Wellenfläche dieselben Hauptschnittebenen schneidet. Die Polarebenen der vier Scheitel sind also Berührungsebenen der Wellenfläche und daher Berührungsebenen einer der entwickelbaren Flächen $\boldsymbol{F}$, welche einer Kugel und der Wellenfläche umschrieben sind. Da nun diese vier Berührungsebenen die Fläche $\boldsymbol{F}$ vollkommen bestimmen, und ebenso die Polarfläche des ellipsoidischen Kegelschnitts, so fallen die beiden Flächen zusammen.

Auf ganz ähnliche Art ergiebt sich der Beweis, dafs die Polarflächen der sphärischen Kegelschnitte die entwickelbaren Flächen $\boldsymbol{G}$ sind.

Es folgt daraus, da/s die Wellenfläche in Beziehung auf das Ellipsoid $D$ ihre eigene Polarfläche ist; d. h. dafs sie die Einhüllungsfläche der Polarebenen ihrer Punkte in Beziehung auf $\boldsymbol{D}$ ist $\left.{ }^{*}\right)$.

*) Ein Satz, welcher bereits von Herrn Plïclier (Bd. 19 S. 42 dieses Journals) aufgestellt worden ist. 


\section{1.}

Die zwei entwickelbaren Flächen $\boldsymbol{F}$ und $\boldsymbol{G}$, welche eine bestimmte Berührungsebene mit der Wellenfläche gemein haben, werden von dieser Berührungsebene längs zweier Erzeugenden berührt, welche Tangenten an die Krümmungslinien durch den Berührungspunkt auf der Wellenfläche sind; und die Berührungskurven der entwickelbaren Flächen $F$ und $G$ mit der Wellenfläche sind die Krümmungslinien der Wellenfläche.

Denn es sei wieder $\boldsymbol{O N}$ gemeinschaftliche Mantellinie zweier sich rechtwinklig schneidender Kegel $\boldsymbol{K}_{1}$ und $\boldsymbol{K}_{2}$, welche die optischen Axen von $\boldsymbol{E}$ zu Fokallinien haben, $\boldsymbol{T}$ eine der auf $\boldsymbol{O N}$ senkrechten Berührungsebenen der Wellenfläche. $\boldsymbol{T}$ ist Berührungsebene einer entwickelbaren Fläche $\boldsymbol{F}_{\mathfrak{l}}$ und einer entwickelbaren Fläche $\boldsymbol{G}_{2}$, von denen die erste durch den Kegel $\boldsymbol{K}_{1}$, die zweite durch den Kegel $\boldsymbol{K}_{2}$ bestimmt ist (9.). Die Erzeugende von $\boldsymbol{F}_{1}$ in $\boldsymbol{T}$ liegt in der Normalebene von $\boldsymbol{K}_{1}$ längs $\boldsymbol{O N}$, die Erzeugende von $\boldsymbol{G}_{2}$ in $\boldsymbol{T}$ liegt in der Normalebene von $\boldsymbol{K}_{2}$ längs $\boldsymbol{O N}$. Geht man von der gemeinschaftlichen Mantellinie $O N$ weiter zu der ihr unendlich nahen Mantellinie auf $\boldsymbol{K}_{2}$ und betrachtet man diese als Mantellinie eines neuen Kegels $\boldsymbol{K}_{1}$, so erhält man senkrecht zu ihr eine Berührungsebene einer neuen Fläche $\boldsymbol{F}_{1}$, welche auch auf der Normalebene zum ersten Kegel $\boldsymbol{K}_{1}$ längs $O N$ senkrecht steht. Die Erzeugenden, längs welcher die zwei Flächen $\boldsymbol{F}$ von den zwei aufeinanderfolgenden Berührungsebenen berührt werden, liegen in der Normalebene und die Schnittlinie der zwei aufeinanderfolgenden Berührungsebenen oder die Tangente an die Berührungskurve der Fläche $\boldsymbol{F}$ mit der Wellenfläche steht senkrecht auf der Normalebene zu $K_{1}$ längs $O N$. Ganz ähnlich ergiebt sich, dafs die Tangente an die Berührungskurve von $\boldsymbol{G}_{2}$ mit der Wellenfläche senkrecht steht auf der Normalebene zu $K_{2}$ längs $\boldsymbol{O N}$.

Die Berührungskurven der Flächen $\boldsymbol{F}$ und $\boldsymbol{G}$ mit der Wellenfläche durchschneiden sich also rechtwinklig und in jedem Punkt der Berührungskurve ist die Erzeugende der einen Fläche Tangente an die Berührungskurve der andern.

Ist nun $O N$ Mantellinie eines Kegels $K_{1}$ und $O S$ die entsprechende Mantellinie des Ergänzungskegels $\boldsymbol{C}_{1}, \boldsymbol{R}^{\prime} \boldsymbol{M}^{\prime}$ die Spur der (auf der Ebene $\mathbf{S O N}$ senkrechten) Polarebene von $\boldsymbol{S}$ auf $\boldsymbol{E}$ für eine Kugel mit dem Mittelpunkt $\boldsymbol{O}$ und mit der Längeneinheit als Halbmesser, so ist (wie sich aus (4.) ergiebt) $\boldsymbol{R}^{\prime} \boldsymbol{M}^{\prime}$ die Richtung der Normale zur Wellenfläche, welche zu der auf $\boldsymbol{O N}$ 
76 7. P. Zech, dic Krïmmungslinien der Wellenflüche zweiaxiger Krystalle.

senkrechten Berührungsebene $\boldsymbol{T}$ im Abstand $\frac{1}{O S}$ von $\boldsymbol{O}$ gehört. Die Linie $\boldsymbol{R}^{\prime} \boldsymbol{M}^{\prime}$ ist (4.) gemeinschaftliche Tangente an das Ellipsoid $\boldsymbol{E}$ und eine concentrische Kugel vom Halbmesser $\frac{1}{O S}$, also Erzeugende einer entwickelbaren Fläche $\boldsymbol{H}$, der Einhüllungsfläche der dem Ellipsoid und der Kugel gemeinschaftlichen Berührungsebenen. Ist $s$ unendlich nahe an $\boldsymbol{S}$ auf dem sphärischen Kegelschnitt, in welchem $\boldsymbol{C}_{1}$, das Ellipsoid $\boldsymbol{E}$ schneidet, so erhält man eine neue Erzeugende $r^{\prime} m^{\prime}$ der Fläche $\boldsymbol{H}$, welche die vorhergehende schneidet und mit ihr die auf SON senkrechte Berührungsebene der Fläche $\boldsymbol{H}$ bestimmt, die Polarebene von $\boldsymbol{S}$ für die Kugel mit der Längeneinheit als Halbmesser. Ist $\boldsymbol{R}$ der Berührungspunkt der Wellenfläche mit $\boldsymbol{T}$, $\boldsymbol{r}$ der Berührungspunkt der Wellenfläche mit der neuen Berührungsebene $t$ an die Wellenfläche, die sich aus $\boldsymbol{O}$ s ergiebt, so ist $\boldsymbol{R} \boldsymbol{r}$ ein Element der Berührungskurve einer Fläche $\boldsymbol{F}$ mit der Wellenfläche, also senkrecht zur Ebene $\boldsymbol{S O N}$, der Normalebene des Kegels $K_{1}$ längs $O N$. Denkt man sich also durch $R r$ eine Ebene parallel zur Ebene $\boldsymbol{R}^{\prime} \boldsymbol{M}^{\prime} \boldsymbol{m}^{\prime} \boldsymbol{r}^{\prime}$, so fallen die Parallelen zu $\boldsymbol{R}^{\prime} \boldsymbol{M}^{\prime}$ durch $\boldsymbol{R}$ und zu $\boldsymbol{r}^{\prime} \boldsymbol{m}^{\prime}$ durch $\boldsymbol{r}$ in diese Ebene, d. h. die Normalen in $\boldsymbol{R}$ und $\boldsymbol{r}$ zur Wellenfläche schneiden sich. Die aufeinanderfolgenden Normalen der Wellenfläche längs der Berührungskurve einer Fläche $\boldsymbol{F}$ mit der Wellenfläche schneiden sich, diese Berührungskurven sind Krümmungslinien der Wellenfläche, also auch die sie rechtwinklig schneidenden Berührungskurven der Flächen $\boldsymbol{G}$.

Da die Wellenfläche Einhüllungsfläche der Flächen $\boldsymbol{F}$ oder der Flächen $\boldsymbol{G}$ ist, so kann man auch sagen, die Charakteristiken bei diesen zwei Einhüllungsarten seien die Krümmungslinien der Wellenfläche.

Nur die eine Reihe Krümmungslinien (die auf den Flächen $\boldsymbol{H}^{\prime}$ ) wird durch folgende Definition bestimmt: Eine Krümmungslinie der Wellenfläche ist der geometrische Ort der Berührungspunkte aller sich gleich schnell fortpflanzender ebener Wellen mit der Wellenfläche. Darunter befinden sich die Berührungskreise der singulären Berührungsebenen.

Stuttgart, März 1857. 